\title{
Prognostic role of $K R A S$ mutations in Sardinian patients with colorectal carcinoma
}

\author{
GRAZIA PALOMBA $^{1 *}$, ANTONIO COSSU $^{2 *}$, PANAGIOTIS PALIOGIANNIS $^{2 *}$, \\ ANTONIO PAZZOLA ${ }^{3}$, GIOVANNI BALDINO ${ }^{4}$, MARIO SCARTOZZI $^{5}$, MARIA TERESA IONTA $^{5}$, \\ SALVATORE ORTU $^{6}$, FRANCESCA CAPELLI $^{7}$, ANNAMARIA LANZILLO ${ }^{8}$, TITO SEDDA ${ }^{9}$, \\ GIOVANNI SANNA ${ }^{10}$, MICHELA BARCA ${ }^{11}$, LUCIANO VIRDIS ${ }^{12}$, MARIO BUDRONI $^{2}$ and GIUSEPPE PALMIERI ${ }^{1}$ \\ ${ }^{1}$ Institute of Biomolecular Chemistry, CNR; ${ }^{2}$ Department of Surgical, \\ Microsurgical and Medical Sciences, University of Sassari; ${ }^{3}$ Oncology Unit, Local Health Unit (ASL1), 07100 Sassari; \\ ${ }^{4}$ Oncology Unit, Civil Hospital, 07041 Alghero; ${ }^{5}$ Department of Medical Oncology, University of Cagliari, 09042 Cagliari; \\ ${ }^{6}$ Oncology Unit, Local Health Unit (ASL2), 07026 Olbia; ${ }^{7}$ Oncology Unit, Zonchello Hospital, 08100 Nuoro; \\ ${ }^{8}$ Oncology Unit, Businco Hospital, 09121 Cagliari; ${ }^{9}$ Oncology Unit, Local Health Unit (ASL), 09170 Oristano; \\ ${ }^{10}$ Department of Medical Oncology, Hospital University (AOU), 07100 Sassari; ${ }^{11}$ Oncology Unit, Local Health Unit (ASL4), \\ 08045 Lanusei; ${ }^{12}$ Oncology Unit, Local Health Unit (ASL7), 09013 Carbonia-Iglesias, Italy
}

Received August 2, 2015; Accepted May 16, 2016

DOI: $10.3892 / 01.2016 .4798$

\begin{abstract}
The presence of mutations in the KRAS gene is a predictor of a poor clinical response to EGFR-targeted agents in patients affected by colorectal cancer (CRC), but its significance as a global prognostic factor remains unclear. The aim of the present study was to evaluate the impact of the KRAS mutational status on time to first metastasis (TTM) and overall survival (OS) in a cohort of Sardinian CRC patients. A total of 551 patients with metastatic CRC at the time of enrolment were included. Clinical and pathological features of the disease, including follow-up information, were obtained from medical records and cancer registry data. For mutational analysis formalin-fixed paraffin-embedded tissue samples were processed using a standard protocol. The coding sequence and splice junctions of exons 2 and 3 of the KRAS gene were screened for mutations by direct automated sequencing. Overall, 186 KRAS mutations were detected in 183/551 (33\%) patients: 125 (67\%) were located in codon $12,36(19 \%)$ in codon 13 , and $18(10 \%)$ in codon 61 . The remaining mutations (7; $4 \%)$ were detected in uncommonly-affected codons. No significant correlation between KRAS mutations and gender, age, anatomical location and stage of the disease at the time
\end{abstract}

Correspondence to: Dr Panagiotis Paliogiannis, Department of Surgical, Microsurgical and Medical Sciences, University of Sassari, 43B Viale San Pietro, 07100 Sassari, Italy

E-mail: ppaliogiannis@uniss.it

*Contributed equally

Key words: KRAS, mutations, colorectal, cancer, target therapies, metastasis, survival of diagnosis was identified. Furthermore, no prognostic value of KRAS mutations was found considering either TTM or OS. When patients were stratified by KRAS mutational status and gender, males were significantly associated with a longer TTM. The results of the present study indicate that KRAS mutation correlated with a slower metastatic progression in males with CRC from Sardinia, irrespective of the age at diagnosis and the codon of the mutation.

\section{Introduction}

Colorectal cancer (CRC) is the third most common neoplastic disease in males, after lung and prostate cancer, and the second in females after breast cancer; furthermore, it is the fourth most frequent neoplastic cause of mortality after lung, stomach, and liver cancer (1). Excellent results have been obtained in the management of non-invasive and early-stage invasive disease in recent times, particularly due to improvements in multidisciplinary endoscopic, surgical, radiotherapeutic and medical oncology protocols. Conversely, progression in the setting of advanced and/or metastatic disease have been less consistent, and relative survival rates remain low (1-3). The role of tumour genetics in this setting is extremely important, particularly in the development of novel therapeutic strategies, based on specific molecular targets.

The most relevant results of 'targeted therapies' have been obtained thanks to the comprehension of the pathophysiological mechanisms involving the epidermal growth factor receptor (EGFR)-RAS cascade (4). The EGFR is a trans-membrane protein for epidermal growth factor that mediates its functions through the activation of the RAS protein family members (HRAS, KRAS, and NRAS) (4). Activated RAS proteins promote cell proliferation through a number of mechanisms, including constitutive stimulation of mitogen activated protein-kinases (MAPK) (4). EGFR-targeted agents 
that compete with EGF for binding the receptor have been employed in clinical practice, in order to inhibit cell proliferation (5). Oncogenic RAS activation is a result of specific mutations into the kinase regions of the genes, producing a constitutive induction of the phosphorylating function of the RAS proteins, which in turn promotes neoplastic proliferation and drastically reduces the effect of anti-EGFR therapies $(5,6)$. Indeed, mutations in KRAS have been extensively demonstrated to act as a predictor of the absence of clinical response to EGFR-targeted agents (7-9). Nevertheless, the significance of KRAS mutation as a global prognostic factor of disease progression and survival in patients with colorectal cancer is not clear. Previous studies have reported a statistically significant reduction in disease free (DFS) and overall survival (OS) in the presence of KRAS mutations $(10,11)$. Nevertheless, such findings were not confirmed in another study (12). The aim of the present study was to evaluate the significance of the KRAS mutational status in terms of time to first metastasis (TTM) and OS in a cohort of Sardinian patients with CRC.

\section{Materials and methods}

Study population. A total of 551 patients with a histologically proven diagnosis of metastatic CRC at the time of enrolment, who were treated and followed-up at healthcare institutions across Sardinia, were included into the study. To avoid any bias, CRC patients were consecutively collected from March 2009 to July 2014; they were included regardless of age at diagnosis and disease characteristics. Cases associated with syndromic colorectal polyposis were excluded.

Sardinian origin was ascertained in all cases through genealogical studies; for all patients, place of birth of their parents and grandparents was assessed in order to assign their geographical origin within the island. Clinical and pathological features for the assessment of the disease stage at diagnosis as well as of the onset age and tumor anatomical location were confirmed by medical records and/or pathology reports. Follow-up and prognostic information were obtained from clinical records at the institutions participating to the study and the records from the local cancer registry. The local cancer registry collects data on neoplastic diseases affecting inhabitants in north Sardinia, through the local hospitals and health care services as with other registries (e.g., death registries), and forms part of a wider network of tumour registries coordinated by the Italian Association for Tumour Registries (Associazione Italiana Registri Tumori, AIRTUM).

All patients gave written informed consent for KRAS mutation analysis prior to tissue sample collection. The study was reviewed and approved by the ethics committee of the Local Health Agency of Sassari (Sassari, Italy).

KRAS mutational analysis. Formalin-fixed paraffin embedded tissue samples from CRC patients were obtained from the archives of the Institutes and Services of Pathology participating in the study. Tissue samples were estimated to contain at least $70 \%$ neoplastic cells by light microscopy. Subsequently, all tumor tissues were processed at the laboratory of the Institute of Biomolecular Chemistry of Sassari; genomic DNA was isolated from tissue sections using a standard protocol and DNA quality assessed for each specimen. In particular, paraffin was removed from formalin-fixed paraffin-embedded (FFPE) samples by treatment with Bio-Clear (Bio-optica, Milan, Italy) and DNA was purified using the QIAamp DNA FFPE Tissue kit (QIAGEN Inc., Valencia, CA, USA).

The coding sequence and splice junctions of exons 2, 3, and 4 of the KRAS gene (where all pathogenic mutations occur), were screened for mutations by direct automated sequencing. Briefly, polymerase chain reaction (PCR) was performed on 25-50 ng of isolated genomic DNA in a 9,700 Thermal cycler (Life Technologies/ThermoFisher Scientific, Inc., Waltham, MA, USA); all PCR products were directly sequenced using an automated fluorescence-based cycle sequencer (ABIPRISM 3130, Life Technologies, ThermoFisher Scientific Inc.), as previously described (13). Primer sequences and protocols for PCR-based assays were designed and optimized in our laboratory; they are available upon request.

Statistical analysis. Statistical analysis was performed using the statistical package SPSS version 7.5 for Windows (SPSS, Inc., Chicago, IL, USA) and Pearson's Chi-Squared test. Descriptive analysis for qualitative variables was conducted using proportions. $\mathrm{P} \leq 0.05$ was considered to indicate a statistically significant difference. Furthermore, a Cox proportional hazards regression model using the Newton-Raphson algorithm was used to determine the impact of the following variables on survival: Gender, age, KRAS status and mutation codons.

\section{Results}

Paraffin-embedded tumour tissues from a total of 551 patients with invasive CRC and originating from Sardinia island were screened for mutations in the KRAS gene. The median age of the patients was 65 years (range, 32-87 years), with a preponderance of males (328 men; 59.5\%). Considering the location of the primary tumour, the left colon was most frequently involved [left colon, 263 (48\%); right-transverse colon, 181 (29\%); rectum, 107 (19\%)] (Table I). Considering the disease stage at the time of diagnosis, the majority of patients presented without distant metastases [55\%; AJCC stages II and III, 162 (29.4\%) and 140 (25.4\%), respectively] (Table I).

The full coding sequences and intron-exon junctions of the KRAS gene were sequenced in the entire series of 551 CRC patients. Overall, KRAS mutations were detected in 183 (33\%) patients; 3 patients presented with coexistence of 2 KRAS mutations in the same primary tumor tissue (Table II). In terms of the gene positions of the identified 186 KRAS mutations, $125(67 \%)$ of the mutations were located in codon 12, $36(19.4 \%)$ were located in codon 13, and 18 (9.7\%) were in codon 61 (Fig. 1). The remaining mutations $(7 ; 3.8 \%)$ were detected in uncommonly-affected codons of the KRAS gene (Table II and Fig. 2). All KRAS mutations detected in the present study have been previously reported in the Human Gene Mutation Database (HGMD) of the Institute of Medical Genetics in Cardiff (http://archive.uwcm.ac.uk) and in the Catalogue Of Somatic Mutations In Cancer (COSMIC) of the Wellcome Trust Sanger Institute (http://www.sanger.ac.uk/genetics/CGP/cosmic/).

No statistically significant correlations were identified between the presence of KRAS mutations and gender, age at diagnosis, anatomical location of primary CRC, and AJCC stage of the disease at the time of diagnosis (Table I). 
Table I. Distribution of mutations according to the characteristics of colorectal cancer patients.

\begin{tabular}{lcr}
\hline Characteristic & No. $(\mathrm{N}=551)$ & KRAS mutations $(\%)$ \\
\hline Gender & 328 & $103(31)$ \\
Male & 223 & $80(36)$ \\
Female & & \\
Tumor site & 181 & $62(34)$ \\
Right-transverse colon & 263 & $85(32)$ \\
Left colon & 107 & $36(34)$ \\
Rectum & & \\
Disease stage at diagnosis & 162 & $50(31)$ \\
Stage II & 140 & $47(34)$ \\
Stage III & 249 & $86(35)$ \\
Stage IV & & \\
Age, years & 51 & $18(35)$ \\
$<50$ & 122 & $48(39)$ \\
$50-59$ & 200 & $64(32)$ \\
$60-69$ & 178 & $53(30)$ \\
$70+$ & & 0.933 \\
\hline
\end{tabular}

Table II. Unusual KRAS mutations found in the cohort of the present study.

\begin{tabular}{|c|c|c|}
\hline Amino acid mutation & DNA mutation & No. cases \\
\hline \multicolumn{3}{|c|}{ Mutations in uncommon codons } \\
\hline p.G12_G13insAG & c.35_36insAGCTGG & 1 \\
\hline p.E49K & c. $145 \mathrm{G}>\mathrm{A}$ & 1 \\
\hline p.A59E & c. $176 \mathrm{C}>\mathrm{A}$ & 1 \\
\hline p.K117N & c. $351 \mathrm{~A}>\mathrm{T}$ & 1 \\
\hline p.A146T & c. $436 \mathrm{G}>\mathrm{A}$ & 3 \\
\hline \multicolumn{3}{|c|}{ Coexistent multiple mutations } \\
\hline p.G12D+p.Q61H & c. $35 \_36 \mathrm{GT}>\mathrm{AC}+\mathrm{c} .183 \mathrm{~A}>\mathrm{C}$ & 1 \\
\hline p.G12D+p.Q61L & c. $35 \_36 \mathrm{GT}>\mathrm{AC}+\mathrm{c} .182 \mathrm{~A}>\mathrm{T}$ & 1 \\
\hline p.G13V+p.Q61H & c. $38 \mathrm{G}>\mathrm{T}+\mathrm{c} .183 \mathrm{~A}>\mathrm{C}$ & 1 \\
\hline
\end{tabular}

Using the Cox proportional hazards model, no prognostic values of KRAS mutations were identified for the TTM [Odds Ratio (OR) $1.18,95 \%$ CI $0.81-1.72, \mathrm{P}=0.198$ ] or OS (OR 1.28, 95\% CI $0.87-1.90, \mathrm{P}=0.107$ ) (Table IIIA). When patients were stratified by both KRAS mutational status and gender, significantly improved TTM was observed for KRAS-mutated male cases (OR 1.58, 95\% CI 0.96-2.60, $\mathrm{P}=0.035$ ) (Table IIIA; Fig. 3A). Conversely, no difference in OS was observed between the two genders according to the occurrence of $K R A S$ mutations (Fig. 3B). Considering the gene positions of the identified KRAS mutations, no correlation with both the TTM (Table IIIB) and OS (data not shown) was observed.

\section{Discussion}

The KRAS proto-oncogene encodes a guanosine triphosphate (GTP)/guanosine diphosphate (GDP) binding protein $(21 \mathrm{kDa})$, which regulates cell responses to a number of extracellular stimuli (14). Activating mutations in KRAS are dominant and generally appear in an early stage of CRC carcinogenesis (early-intermediate adenoma), maintaining a stable incidence in higher stages (late adenomas and carcinomas). Approximately 30-50\% of CRC patients have KRAS mutations, and they are associated with poor responsiveness to anti-EGFR antibody therapies $(7-10,15)$. In the present study, $33 \%$ of the patients with CRC harboured a mutation in the KRAS gene. In accordance with previous reports, no statistically significant differences in the distribution of these mutations between genders, ages, disease locations, and stages were found in our cohort (10). In addition, the mutation sites within the gene were similar to those widely reported, with the codons 12 and 13 affected in $\sim 80 \%$ of cases. Notably, 7 mutations were identified in uncommonly-affected codons (Table II). In total, 6 of these mutations were single base substitutions and one was an insertion between codons 12 and 13 (c.35_36insAGCTGG). $K R A S$ insertions have been sporadically reported among CRC 

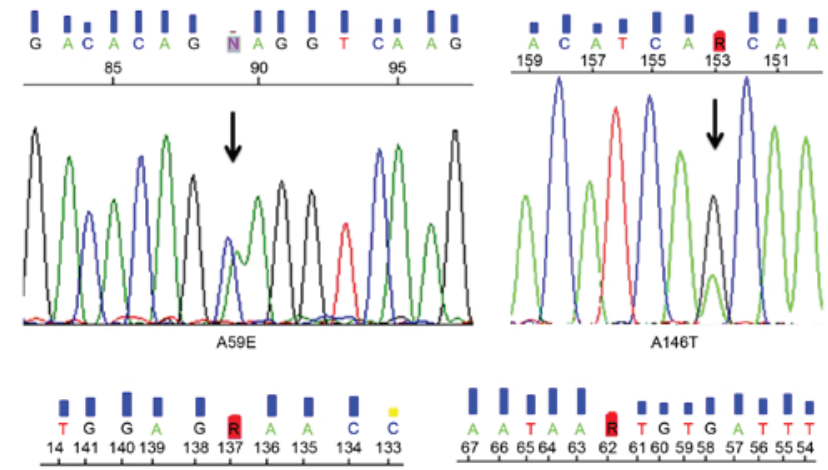

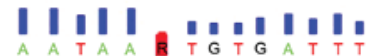

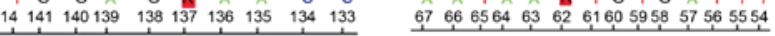
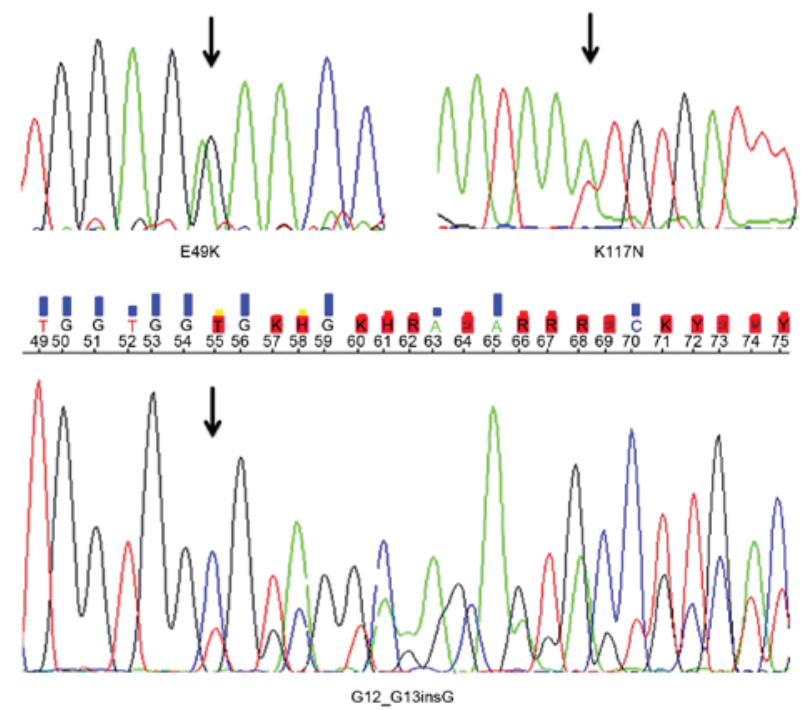

Figure 1. Types and frequencies of the mutations observed in the cohort.

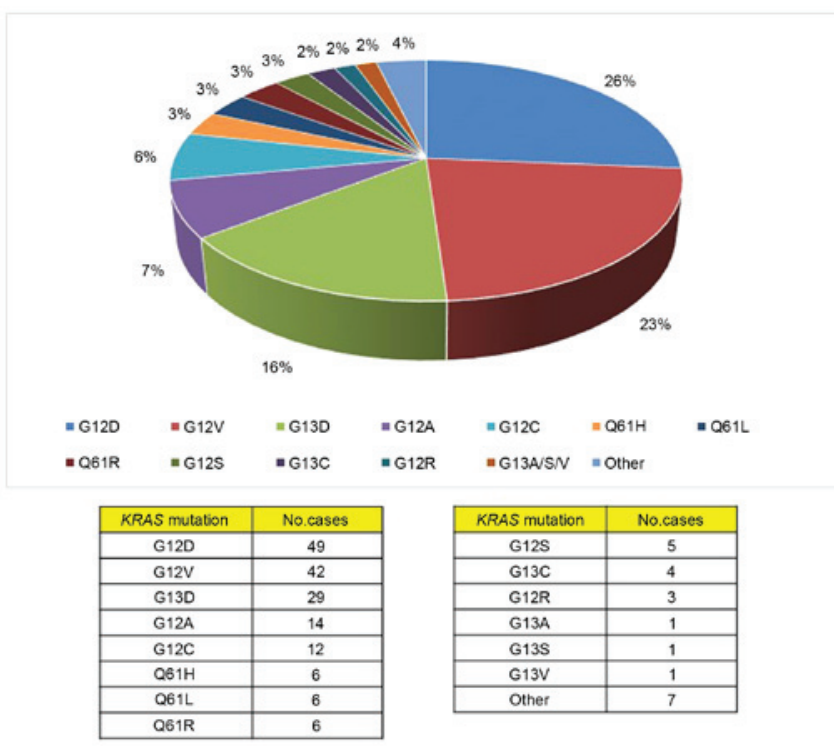

Figure 2. Mutations in uncommonly-affected codons of the KRAS gene found in the cohort.

patients; overall, biological and clinical significance of such mutations is yet to be defined (16).

Despite knowledge of the effect of KRAS mutations in predicting lack of response to anti-EGFR therapy, the global impact of such mutations on OS and DFS in CRC patients
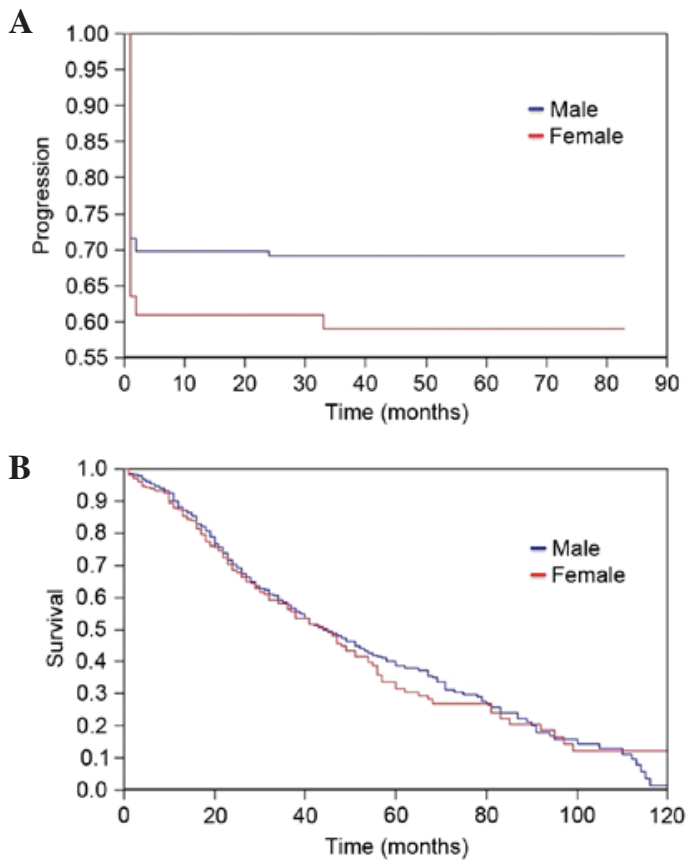

Figure 3. (A) Time to first metastasis and (B) overall survival according to gender in patients harboring KRAS mutations.

remains controversial. In 1994, Tanaka et al (17) published the first valuable article on such a topic. The authors examined a cohort of $15 K R A S$ mutations occurring in 62 Japanese patients using an oligonucleotide hybridization technique, and found that KRAS mutation was an independent prognostic factor in a multivariate analysis (17). In the same year, Morrin et al (18) published a less extensive study on 52 CRC patients with 14 KRAS mutations, without evidence of any prognostic impact. One year later, Bennett et al (19) published their series of 157 Dukes' stage B CRCs with mutations in codon 12, which were analysed by PCR and direct sequencing. The authors failed to demonstrate a predictive role for survival of KRAS mutations by $\log$ rank and multivariate analysis (19). Lee et al (20) subsequently reported a series of 64 Chinese patients; 38 patients were Dukes' stage A and B, and 26 patients were Dukes' stage C and D (20). Log rank tests identified that KRAS gene mutation and Dukes' stage were associated with patients' survival; furthermore, patients with $K R A S$ mutations were found to have shorter survival times into the subgroup with Dukes' stage A and B, but not in that with stages C and D (20). Pricolo et al (21) published their series on 70 Dukes' stage $\mathrm{C}$ patients analysed by topographic genotyping, and found a $36 \%$ KRAS mutations that did not affect significantly the risk of mortality or the 5-year survival rate. In 1998, Kressner et al (22) published their results on a series of 62 KRAS mutations found by temporal temperature gradient gel electrophoresis among 191 Swedish CRC patients; again, KRAS mutations had no relevant prognostic importance (22). Conversely, Andreyev et al (23) performed the RASCAL study, a large cohort collaborative report which indicated that KRAS mutations-especially those of codon 12 were associated with increased risk of relapse or mortality. More specifically, the subsequent RASCAL II study, which involved 3,498 patients with CRC, indicated that only the 
Table III. Correlation between KRAS mutational status and survival.

A, KRAS mutation status in toto

\begin{tabular}{lccc}
\hline & & \multicolumn{2}{c}{ Confidence interval } \\
\cline { 3 - 4 } Parameter & Odds Ratio & Lower & Upper \\
\hline TTM & & & 0.198 \\
KRAS status & 1.187 & 0.816 & 0.254 \\
Female and $K R A S$ status & 0.799 & 0.444 & 0.035 \\
Male and $K R A S$ status & 1.583 & 0.962 & 1.439 \\
OS & & & 2.606 \\
$K R A S$ status & 1.286 & 0.874 & 0.107 \\
Female and $K R A S$ status & 1.151 & 0.825 & 0.100 \\
Male and $K R A S$ status & 1.141 & 0.679 & 2.766 \\
\hline
\end{tabular}

B, KRAS codon-specific mutations

\begin{tabular}{|c|c|c|c|c|}
\hline \multirow[b]{2}{*}{ Parameter } & \multirow[b]{2}{*}{$\mathrm{P}$-value } & \multirow[b]{2}{*}{ Hazard ratio } & \multicolumn{2}{|c|}{ Confidence interval } \\
\hline & & & Lower & Upper \\
\hline \multicolumn{5}{|l|}{ TTM } \\
\hline Gender & 0.011 & 0.728 & 0.569 & 0.931 \\
\hline Age at diagnosis & 0.473 & 1.161 & 0.771 & 1.750 \\
\hline Codon 12 mutations & 0.907 & 1.037 & 0.559 & 1.924 \\
\hline Codon 13 mutations & 0.861 & 1.045 & 0.634 & 1.721 \\
\hline Other codon mutations & 0.806 & 1.076 & 0.598 & 1.935 \\
\hline
\end{tabular}

TTM, time to first metastasis; OS, overall survival.

substitution of glycine to valine on codon 12 had a significant influence on OS, particularly among patients with Dukes' stage C carcinomas (10). These studies did not indicate any prognostic role for mutations in codon 13 of the KRAS gene. Certain authors consider that the prognostic value for the $\mathrm{KRAS}^{\mathrm{G} 12 \mathrm{~V}}$ mutation in the RASCAL study may represent the result of methodological limitations due to the retrospective recollection of patients enrolled in different studies using 3 different mutational tests, and possible nonrandom systemic errors that may affect meta-analysis of such datasets (24).

In 2000, Samowitz et al (25) published their results on a large cohort including $100 \mathrm{KRAS}^{\text {codon } 13}$ mutations identified by PCR and direct sequencing-in 1,413 CRC patients from California and Utah. The authors found that generally KRAS mutations were not associated with increased cancer-associated mortality in their cohort; nevertheless, mutations in codon 13 were associated with a $40 \%$ greater likelihood of dying, although the significance of such finding was borderline when adjusted for age and disease stage (25). Other valuable studies on the prognostic significance of $K R A S$ mutations in CRC patients were performed during the 2000s; the most relevant series were those of Ogino et al (26) in the USA, Zlobec et al (27) in Switzerland, Winder et al (28) in Austria, and Bazan et al (29) in Italy. Ogino and collegues performed an evaluation of the independent effect of the $\mathrm{CpG}$ island methylator phenotype (CIMP), microsatellite instability (MSI), KRAS and $B R A F$ mutations on the prognosis of 649 patients with I-IV stage CRC. The study did not identify a relevant role of the KRAS mutations on prognosis, but the authors provided evidence that CIMP-high appears to be an independent predictor of a low CRC-specific mortality, while $B R A F$ mutations are associated with a high CRC-specific mortality (26). By contrast, in their combined analysis of specific KRAS gene alterations, $\mathrm{BRAF}^{\mathrm{V} 600 \mathrm{E}}$ status, and MSI, Zlobec et al (27) found that specific KRAS mutations act as informative prognostic factors in both sporadic and hereditary CRC; an algorithm including all these alteration may identify sporadic CRC patients with poor clinical outcome. Winder et al (28) in their Austrian series of 342 cases were the first to report an improved OS in patients with $\mathrm{KRAS}^{\text {codon } 13}$ mutations compared to those with wild-type genes, further suggesting that different types of KRAS mutations may be differently associated with OS in patients with CRC (28). Finally, a multivariate analysis by Bazan et al (29) in a series of 160 cases found that KRAS ${ }^{\text {codon } 13}$ mutations were associated with poorer OS but not DFS (29).

It is evident that the global prognostic impact of $K R A S$ mutations in patients with colorectal cancer remains unclear. The discrepancy of results among the studies mentioned 
may be explained on the basis of the relevant number of confounding and heterogeneity factors. The most frequent are: a) Heterogeneity of the study populations; b) discrepancies in study design and methodology; c) strategies for detecting $K R A S$ mutations (including type of tissue samples i.e., primary or metastatic tumours); d) presence of underlying predisposing conditions (i.e. ulcerative colitis); e) variability of the staging systems (Dukes, AJCC, different editions); and f) clinical management and treatments employed.

Ren et al (30) have recently published a well-designed review and meta-analysis on the impact of KRAS mutations on OS in CRC patients, with the aim to reduce biases as those mentioned above (30). The authors performed a careful selection of 23 articles published from 1992, including 25 sets of data with 4,687 patients and 1,364 (29\%) KRAS-mutated cases. Among them, 9 indicated that KRAS mutations were associated with worse prognosis, 15 failed to demonstrate any statistically significant association between such mutations and prognosis, and, finally, one data set identified an improved survival rate for patents with CRC and KRAS mutations (30). Globally, the authors found that KRAS mutations were not associated with CRC prognosis, either before or after adjustment for the effect of publication bias. Notably, the authors found that KRAS mutations in codon 13, and the technique of mutation detection (PCR-RFLP or PCR-SSCP, followed by sequencing) were associated with poorer OS; nevertheless, the authors considered that sample size may have not enough statistical power to accurately detect the association between KRAS mutations and survival (30).

In the present study, a clear association between occurrence of KRAS mutations and TTM or OS was not demonstrated in the entire series of 551 CRCs. When patients were stratified by both KRAS mutational status and gender, again, no significant differences in OS were identified, but a significantly improved TTM was observed for KRAS-mutated male cases. This finding is particularly notable, as the present study is the first to describe it in a series with a consistent number of CRC patients. Furthermore, TTM was determined which in our opinion better describes the biological events of CRC evolution in comparison with disease free survival (DFS) which better describes the impact of therapies on the progression of the disease.

On this regard, Samowitz et al (25) suggested that male patients are more likely to present transition mutations and $\mathrm{G} \rightarrow \mathrm{A}$ mutations due to different lifestyle factors, including alcohol and tobacco use, or dietary habits, which men and women are differently exposed to. In addition, the hormonal status has been involved in the pathogenesis of CRC through its impact on bile production and composition, bowel transit times, and bacterial metabolism $(25,31)$. A smaller increase in mortality in patients with transition mutations has been previously reported, while the variant with the higher likelihood of mortality was the $\mathrm{G} \rightarrow \mathrm{A}$ mutation at the codon 13 (25). The latter finding was also observed by Cerrottini et al (32). These data evidence that male patients may harbour less aggressive $K R A S$ mutations, and this may explain the findings of the present study; nevertheless, it is not clear why this effect is limited to the initial progression as metastatic disease and does not affect OS. Unfortunately, the issue is not adequately studied in the current literature, and further well-designed studies are necessary to improve the understanding of such a specific prognostic variation in subgroups of patients with KRAS mutations.

The data presented in the current study suggest that KRAS mutations are not generally associated with TTM and OS in patients with CRC. Nevertheless, an improved TTM was observed in male patients with CRC harbouring KRAS mutations. Such a finding may be associated with the specific types of KRAS mutations observed in males and females as consequence of exposure to different lifestyle factors. Further well-designed studies are necessary to confirm such findings.

\section{References}

1. Palmieri G, Paliogiannis P, Scognamillo F, Budroni M, Cesaraccio R, Pulighe F, Tanda F, Trignano M and Cossu A: Colorectal cancer epidemiology in an area with a spontaneous screening program. Acta Med Mediterr 29: 231-234, 2013.

2. Edwards BK, Ward E, Kohler BA, Eheman C, Zauber AG, Anderson RN, Jemal A, Schymura MJ, Lansdorp-Vogelaar I, Seeff LC, et al: Annual report to the nation on the status of cancer, 1975-2006, featuring colorectal cancer trends and impact of interventions (risk factors, screening, and treatment) to reduce future rates. Cancer 116: 544-573, 2010 .

3. Paliogiannis P, Cossu A, Tanda F, Palmieri G and Palomba G: KRAS mutational concordance between primary and metastatic colorectal adenocarcinoma. Oncol Lett 8: 1422-1426, 2014.

4. Jorissen RN, Walker F, Pouliot N, Garrett TP, Ward CW and Burgess AW: Epidermal growth factor receptor: Mechanisms of activation and signalling. Exp Cell Res 284: 31-53, 2003.

5. Yuan ZX, Wang XY, Qin QY, Chen DF, Zhong QH, Wang L and Wang JP: The prognostic role of BRAF mutation in metastatic colorectal cancer receiving anti-EGFR monoclonal antibodies: A meta-analysis. PLoS One 8: e65995, 2013.

6. Malumbres $\mathrm{M}$ and Barbacid M: RAS oncogenes: The first 30 years. Nat Rev Cancer 3: 459-465, 2003.

7. Heinemann V, Stintzing S, Kirchner T, Boeck S and Jung A: Clinical relevance of EGFR- and KRAS-status in colorectal cancer patients treated with monoclonal antibodies directed against the EGFR. Cancer Treat Rev 35: 262-271, 2009.

8. Bardelli A and Siena S: Molecular mechanisms of resistance to cetuximab and panitumumab in colorectal cancer. J Clin Oncol 28: 1254-1261, 2010.

9. Lin AY, Buckley NS, Lu AT, Kouzminova NB and Salpeter SR: Effect of KRAS mutational status in advanced colorectal cancer on the outcomes of anti-epidermal growth factor receptor monoclonal antibody therapy: A systematic review and meta-analysis. Clin Colorectal Cancer 10: 63-69, 2011.

10. Andreyev HJ, Norman AR, Cunningham D, Oates J, Dix BR, Iacopetta BJ, Young J, Walsh T, Ward R, Hawkins $\mathrm{N}$, et al: Kirsten ras mutations in patients with colorectal cancer: The 'RASCAL II' study. Br J Cancer 85: 692-696, 2001.

11. Imamura Y, Morikawa T, Liao X, Lochhead P, Kuchiba A, Yamauchi M, Qian ZR, Nishihara R, Meyerhardt JA, Haigis KM, et al: Specific mutations in KRAS codons 12 and 13 , and patient prognosis in 1075 BRAF wild-type colorectal cancers. Clin Cancer Res 18: 4753-4763, 2012.

12. Phipps AI, Buchanan DD, Makar KW, Win AK, Baron JA, Lindor NM, Potter JD and Newcomb PA: KRAS-mutation status in relation to colorectal cancer survival: The joint impact of correlated tumour markers. Br J Cancer 108: 1757-1764, 2013.

13. Palomba G, Colombino M, Contu A, Massidda B, Baldino G, Pazzola A, Ionta M, Capelli F, Trova V, Sedda T, et al: Prevalence of KRAS, BRAF, and PIK3CA somatic mutations in patients with colorectal carcinoma may vary in the same population: Clues from Sardinia. J Transl Med 10: 178, 2012.

14. Schubbert S, Shannon K and Bollag G: Hyperactive Ras in developmental disorders and cancer. Nat Rev Cancer 7: 295-308, 2007.

15. Arrington AK, Heinrich EL, Lee W, Duldulao M, Patel S, Sanchez J, Garcia-Aguilar J and Kim J: Prognostic and predictive roles of KRAS mutation in colorectal cancer. Int J Mol Sci 13: 12153-12168, 2012. 
16. de Macedo MP, de Lima LG, Begnami MD, de Melo FM, Andrade LD, Lisboa BC, Soares LM, Soares FA, Carraro DM and da Cunha IW: KRAS insertions in colorectal cancer: What do we know about unusual KRAS mutations? Exp Mol Pathol 96: 257-260, 2014

17. Tanaka M, Omura K, Watanabe Y, Oda Y and Nakanishi I: Prognostic factors of colorectal cancer: K-ras mutation, overexpression of the p53 protein, and cell proliferative activity. J Surg Oncol 57: 57-64, 1994.

18. Morrin M, Kelly M, Barrett N and Delaney P: Mutations of Ki-ras and p53 genes in colorectal cancer and their prognostic significance. Gut 35: 1627-1631, 1994.

19. Bennett MA, Kay EW, Mulcahy H, O'flaherty L, O'donoghue DP, Leader $\mathrm{M}$ and Croke DT: Ras and p53 in the prediction of survival in Dukes' stage B colorectal carcinoma. Clin Mol Pathol 48 M310-M315, 1995.

20. Lee JC, Wang ST, Lai MD, Lin YJ and Yang HB: K-ras gene mutation is a useful predictor of the survival of early stage colorectal cancers. Anticancer Res 16: 3839-3844, 1996.

21. Pricolo VE, Finkelstein SD, Wu TT, Keller G, Bakker A Swalsky PA and Bland KI: Prognostic value of TP53 and K-ras-2 mutational analysis in stage III carcinoma of the colon. Am J Surg 171: 41-46, 1996

22. Kressner U, Bjørheim J, Westring S, Wahlberg SS, Påhlman L, Glimelius B, Lindmark G, Lindblom A and Børresen-Dale AL: $\mathrm{Ki}$-ras mutations and prognosis in colorectal cancer. Eur J Cancer 34: 518-521, 1998.

23. Andreyev HJ, Norman AR, Cunningham D, Oates JR and Clarke PA: Kirsten ras mutations in patients with colorectal cancer: The multicenter 'RASCAL' study. J Natl Cancer Inst 90: 675-684, 1998.

24. Roth AD, Tejpar S, Delorenzi M, Yan P, Fiocca R, Klingbiel D, Dietrich D, Biesmans B, Bodoky G, Barone C, et al: Prognostic role of KRAS and BRAF in stage II and III resected colon cancer: Results of the translational study on the PETACC-3, EORTC 40993, SAKK 60-00 trial. J Clin Oncol 28: 466-474, 2010.
25. Samowitz WS, Curtin K, Schaffer D, Robertson M, Leppert M and Slattery ML: Relationship of Ki-ras mutations in colon cancers to tumor location, stage, and survival: A population-based study. Cancer Epidemiol Biomarkers Prev 9: 1193-1197, 2000

26. Ogino S, Nosho K, Kirkner GJ, Kawasaki T, Meyerhardt JA, Loda M, Giovannucci EL and Fuchs CS: CpG island methylator phenotype, microsatellite instability, BRAF mutation and clinical outcome in colon cancer. Gut 58: 90-96, 2009.

27. Zlobec I, Kovac M, Erzberger P, Molinari F, Bihl MP, Rufle A, Foerster A, Frattini M, Terracciano L, Heinimann K and Lugli A: Combined analysis of specific KRAS mutation, BRAF and microsatellite instability identifies prognostic subgroups of sporadic and hereditary colorectal cancer. Int J Cancer 127: 2569-2575, 2010.

28. Winder T, Mündlein A, Rhomberg S, Dirschmid K, Hartmann BL, Knauer M, Drexel H, Wenzl E, De Vries A and Lang A: Different types of K-Ras mutations are conversely associated with overall survival in patients with colorectal cancer. Oncol Rep 21: 1283-1287, 2009

29. Bazan V, Agnese V, Corsale S, Calò V, Valerio MR, Latteri MA, Vieni S, Grassi N, Cicero G, Dardanoni G, et al: Specific TP53 and/or Ki-ras mutations as independent predictors of clinical outcome in sporadic colorectal adenocarcinomas: Results of a 5-year Gruppo Oncologico dell'Italia Meridionale (GOIM) prospective study. Ann Oncol 16 (Suppl 4): iv50-iv55, 2005.

30. Ren J, Li G, Ge J, Li X and Zhao Y: Is K-ras gene mutation a prognostic factor for colorectal cancer: A systematic review and meta-analysis. Dis Colon Rectum 55: 913-923, 2012.

31. Troungos C, Valavanis C, Kapranos N and Kittas C: K-ras mutation in Greek patients with poorly and moderately differenciated tumours of the lower intestinal tract. Anticancer Res 17: 1399-1404, 1997.

32. Cerottini JP, Caplin S, Saraga E, Givel JC and Benhattar J: The type of K-ras mutation determines prognosis in colorectal cancer. Am J Surg 175: 198-202, 1998. 